\title{
Implementação de Estratégias para a Redução da Mortalidade Materna no Vale do Jequitinhonha ${ }^{1}$
}

\author{
Helisamara Mota Guedes ${ }^{2}$ \\ Naila dos Santos Cunha ${ }^{3}$ \\ Juliana Augusta Dias ${ }^{4}$ \\ George Sobrinho Silva ${ }^{5}$
}

Visando contribuir com a redução da mortalidade materna no Vale do Jequitinhonha foi desenvolvido ações com o objetivo de atualizar e uniformizar as condutas clínicas dos profissionais de saúde para a assistência às emergências obstétricas. A ação contemplou os municípios da Microrregião de Saúde de Diamantina em quatro etapas: I - atualização do protocolo; II - apresentação dos resultados para a equipe e ajuste; III - criação de parcerias com a Comissão Intergestora Regional, e apresentação da proposta de um curso de atualização e divulgação do protocolo; IV - realização do Curso de Emergências Obstétricas. Foram coletados dados sobre as características do atendimento a parturiente antes e após a realização do curso. Análise descritiva foi procedida. Compareceram ao evento 108 profissionais de saúde. Foram distribuídos 16 cartazes coloridos de seis diferentes fluxogramas de atendimento aos hospitais de cada município. Dos questionários entregues no início e final do curso obteve-se os seguintes resultados: na maioria dos municípios é garantido às parturientes o direito à presença de acompanhante; houve diferença em pacientes atendidos pelo convênio e SUS; o partograma não é utilizado em sete cidades; os métodos não farmacológicos de alívio da dor mais descritos foram a bola, deambulação e massagem. Observou-se falta de algumas medicações necessárias para intercorrências. A proposta apresentou repercussão positiva, houve uma boa adesão e participação dos profissionais. Espera-se que grandes atores estratégicos tenham sido sensibilizados e que mudança da prática obstétrica aconteça. Torna-se necessário incentivar outros eventos desta natureza para que a mortalidade materna na região diminua cada vez mais.

Palavras-chave: Atendimento de urgências; Enfermagem obstétrica; Mortalidade materna.

\footnotetext{
${ }^{1}$ Projeto de Extensão "Curso de Emergências Obstétricas" da Universidade Federal dos Vales do Jequitinhonha e Mucuri (UFVJM), financiado pelo PROEX-2017.

${ }^{2}$ Enfermeira. Doutora em Enfermagem. Docente do Departamento de Enfermagem da Universidade Federal dos Vales do Jequitinhonha e Mucuri (UFVJM).

3 Acadêmica do Curso de Enfermagem da UFVJM. Estagiária do Curso de Especialização em Enfermagem Obstétrica - Rede Cegonha.

${ }^{4}$ Médica. Mestre pelo Programa de Pós Graduação Ensino e Saúde. Docente da Faculdade de Medicina da UFVJM.

${ }^{5}$ Enfermeiro. Doutor em Enfermagem. Docente do Departamento de Enfermagem da UFVJM.
} 


\title{
Implementation of Strategies for Reducing Maternal Mortality in the Valley do Jequitinhonha
}

\begin{abstract}
Aiming to contribute to the reduction of maternal mortality in the Jequitinhonha Valley, actions were developed with the objective of updating and standardizing the clinical practices of health professionals to assist obstetric emergencies. The action included the municipalities of the Diamantina Health Microregion in four stages: I - protocol update; II - presentation of the results to the team and adjustment; III - creation of partnerships with the Regional Interactive Commission, and presentation of the proposal for a course to update and disseminate the protocol; IV - completion of the Obstetric Emergencies Course. Data were collected on the characteristics of parturient care before and after the course. Descriptive analysis was carried out. 108 health professionals attended the event. Sixteen colored posters of six different flow charts were distributed to hospitals in each municipality. From the questionnaires delivered at the beginning and end of the course the following results were obtained: in the majority of municipalities the parturients are guaranteed the right to the presence of companions; there was difference in patients attended by the agreement and SUS; the partograph is not used in seven cities; the nonpharmacological methods of pain relief most described were ball, ambulation, and massage. There was a lack of some medications needed for complications. The proposal had positive repercussion, there was a good adhesion and participation of the professionals. It is expected that great strategic actors have been sensitized and that change in obstetric practice will happen. It is necessary to encourage other events of this nature so that the maternal mortality in the region decreases more and more.
\end{abstract}

Keywords: Emergency care; Obstetric nursing; Maternal mortality.

\section{INTRODUÇÃO}

Com o intuito de reduzir a mortalidade materna mundial foi estabelecido os Objetivos de Desenvolvimento Sustentável (ODS), como uma iniciativa global promovida pelas Nações Unidas, para eliminação da mortalidade materna evitável entre os anos de 2016 a 2030 (ONUBR, 2017). No caso do Brasil, a meta para 2030 é reduzir a mortalidade materna para aproximadamente 20 mortes para cada 100 mil nascidos vivos, considerando a razão oficial de mortalidade materna no Brasil para o ano de 2010 (SOUZA, 2015).

Ainda se observa um acesso desigual aos serviços de saúde e demoras na identificação e manejo das complicações relacionadas à gestação sendo grandes obstáculos para a sobrevivência e o bem-estar de mulheres (e crianças) em todo o mundo. Desta forma, a batalha cotidiana para eliminar as mortes maternas e promover o bem estar das mulheres deve ser compromisso de todos os indivíduos, em instituições grandes e pequenas, nas 
comunidades perto das pessoas, na solidão dos locais que muitas vezes são tão pequenos que torna difícil a construção de uma rede de cuidados próxima (SOUZA, 2015).

O Vale do Jequitinhonha tem apresentado necessidade de melhoria dos índices de mortalidade materna e neonatal da região. O único estudo desta região foi realizado por Dias (2016) no período de 2010 a 2013, ocorreram 12 óbitos maternos nos hospitais do Alto Jequitinhonha, MG. Quanto a etiologia do óbito materno, apresentaram-se as causas obstétricas diretas como as principais causas de mortalidade materna, com destaque em 3 $(25,0 \%)$ da Síndrome Hellp, 2 (16,6\%) descolamento prematuro de placenta, 2 (16,6\%) insuficiência cardíaca, $1(8,3 \%)$ hemorragia pós parto, 1 (8,3\%) eclampsia e $3(25,0 \%)$ causa indeterminada.

No Brasil, $80 \%$ dos óbitos maternos são decorrentes de causas obstétricas diretas com destaque para as hemorragias e as crises hipertensivas da gravidez, enquanto que as causas obstétricas indiretas são responsáveis por apenas 15 a $20 \%$ dos óbitos. Em países desenvolvidos, esta relação é contrária, com as causa obstétricas indiretas sendo as principais responsáveis pelos óbitos. Esses dados nos permitem refletir sobre a qualidade da assistência prestada a mulher durante o período gestacional, parto e puerpério (SANCHES et al., citado por Dias, 2013).

Diante deste fato e da necessidade de homogeneizar condutas de atendimento a gestante em situação de emergência surgiu a intenção de estabelecer estratégias para instrumentar os profissionais de saúde e os serviços da região para melhoria da qualidade do atendimento a gestantes, parturientes e puérperas. O objetivo foi de atualizar o Protocolo de Condutas Clínicas Obstétricas do Hospital Nossa Senhora da Saúde, oferecer um evento para divulgar este protocolo e capacitar habilidades técnicas e teóricas.

\section{Materiais e métodos}

Trata-se de um projeto de intervenção que foi realizado na Maternidade do Hospital Nossa Senhora da Saúde em Diamantina - Minas Gerais.

O estudo abrangeu a microrregião de Saúde de Diamantina-Minas Gerais, composta por 15 municípios, sendo eles: Alvorada de Minas, Carbonita, Coluna, Congonhas do Norte, Couto de Magalhães de Minas, Datas, Diamantina, Felício dos Santos, Gouveia, 
Itamarandiba, Presidente Kubitschek, Santo Antônio do Itambé, São Gonçalo do Rio Preto, Senador Modestino Gonçalves e Serro.

A Irmandade de Nossa Senhora da Saúde é uma associação religiosa de utilidade pública, de duração indeterminada, de fins caritativos beneficentes, sem fins lucrativos, fundada nesta cidade de Diamantina, em 09 de abril de 1901 (HNSS, 2017).

A maternidade possui 15 leitos de alojamento conjunto, 3 pré-parto, parto e pós-parto (PPP), um bloco cirúrgico e uma UTI Neonatal com 10 leitos. O HNSS é referência na atenção à saúde em gestação de Alto Risco para toda a Região Ampliada de Saúde oferecendo suporte para os demais municípios da Região (HNSS, 2017).

O projeto envolveu as seguintes etapas:

1 - O Protocolo de Condutas Clínicas (DIAS, 2017) foi atualizado seguindo as diretrizes do Advanced Life Suport in Obstetrics (ALSO) e de protocolos de instituições de referência no atendimento de gestantes como Hospital Sofia Feldman/BH, Hospital das Clínicas/BH, Clínica Obstétrica FMUSP, além de manuais do Ministério da Saúde. Houve adaptação para a realidade institucional.

2 - O Protocolo de Condutas Clínicas foi apresentado para a equipe de saúde da Maternidade do Hospital Nossa Senhora da Saúde e feito os ajustes sugeridos.

3 - Foi feito uma reunião com a Superintendência Regional de Saúde (SRS) para solicitar apoio para a realização do Curso de Emergências Obstétricas. O curso foi criado a partir da necessidade da excelência no atendimento das emergências obstétricas para melhoria dos índices de mortalidade materna da região. Teve como foco capacitar profissionais médicos e enfermeiros atuantes no atendimento hospitalar de gestantes, além de acadêmicos e residentes. O apoio da SRS consistia na divulgação do curso junto aos gestores da microrregião de saúde. Nesta reunião foi solicitado que a proposta fosse apresentada aos gestores na reunião da Comissão Intergestora Regional (CIR).

4 - A reunião da CIR aconteceu no dia 03 de maio de 2015 em que foram apresentados os dados de mortalidade materna na Região do Vale do Jequitinhonha e a proposta do curso. Foi 
acordado que os gestores se responsabilizariam em enviar médicos e enfermeiros para o curso.

5 - O Curso de Emergências Obstétricas foi registrado como evento de Extensão da ProReitoria de Extensão e Cultura (PROEXC) recebendo o protocolo de número 016.3.081-20. O evento ocorreu no anfiteatro do campus I, localizado na Universidade Federal dos Vales do Jequitinhonha e Mucuri.

O evento foi divulgado na forma impressa e eletrônica, onde foram disponibilizadas 160 vagas para inscrição na página da Even, uma plataforma de gestão de eventos científicos, disponibilizado no endereço eletrônico: https://www.even3.com.br/cursolago. Através dos emails cadastrados enviou-se informações aos inscritos.. O evento contou com um momento teórico sobre os temas listados abaixo por palestrantes com expertise na área de obstetrícia, da região e de outras instituições como o Hospital Sofia Feldman, Hospital das Clínicas, Faculdade de Ciências Médicas, instrutores do Curso ALSO (Advanced Life Suport in Obstetrics), Suporte de Vida em Obstetrícia.

Após discussão teórica, os profissionais da saúde foram divididos e submetidos a oficinas simuladas de baixa e média fidelidade e feito o debriefing. A cada 40 minutos os participantes mudavam de estação. Desta forma, todos tiveram acesso às cinco estações e os grupos foram compostos por 16 pessoas. Considerando que a simulação exigia conhecimentos prévios não foi aberto vagas para estudantes de graduação, exceto os participantes da Liga de Obstetrícia e Ginecologia e da GEGO.

O Conteúdo programático da parte teórica, totalizando 10 horas, abordou os temas: Mortalidade Materna no Alto Jequitinhonha, Estado Fetal não tranquilizador, Crises hipertensivas, Sepse, Trabalho de parto prematuro, Assistência ao Parto, Atendimento pré hospitalar em emergências obstétricas, Hemorragia Puerperal, Apresentação do Balão de Alves.

O conteúdo programático da parte prática, totalizando 5 horas, abordou os temas: parto operatório e parto pélvico, Ressuscitação cardiopulmonar em gestante, Hemorragia, Treinamento B-Lynch, Distocia de ombro e Eclampsia 
Foi disponibilizado na pasta do evento o CD com as diretrizes do parto normal, versão completa e reduzida, artigo sobre distocia de ombro, um vídeo ensinando a fazer o balão de Alves para tamponamento uterino, o protocolo de Condutas Clínicas do HNSS. Além disto, xérox de um kit para se fazer a caixinha de emergência para Eclampsia e para Hemorragia, além do check list de atendimento.

Para os profissionais que trabalham em hospital ou unidade mista foram distribuído cartazes coloridos de check list para ser afixada no posto de enfermagem com as condutas no atendimento de eclampsia, sepse, hemorragia.

Foi aplicado um questionário para avaliação do evento, tanto antes quanto após. O questionário abordava questões referentes ao local de trabalho, boas práticas do parto, além de perguntas diretas relacionadas à temática do evento.

\section{Resultados e Discussão}

Dos 151 participantes que realizaram sua inscrição, 108 compareceram ao evento. Estiveram presentes representantes de diferentes municípios entre eles: Minas Novas, Diamantina, Belo Horizonte, Itumbiara, Turmalina, Itamarandiba, Carbonita, Itaobim, Virgem da Lapa, Alvorada de Minas, Berilo, Araçuaí, Felício dos Santos, Presidente Kubitscheck, Capelinha, Curvelo, Serro, Coluna, Serra Azul de Minas, São Gonçalo do Rio Preto, Senador Modestino Gonçalves. Desta forma, houve representantes de outros municípios do Estado de Minas Gerais, extrapolando a microrregião de Diamantina.

Foram confeccionados 16 cartazes de seis diferentes fluxogramas de atendimento. Cada hospital ganhou um cartaz de cada, não tendo em quantidade suficientes para todos os representantes de todas as cidades que compareceram.

Os médicos e enfermeiros puderam trocar experiência, tirar dúvidas e sentir-se incomodados a indagar sua prática clínica. Teve um grupo que trouxe um aparelho (AMIU) de seu hospital para que os professores orientassem sobre como utilizá-lo. O aparelho não era utilizado por deficiência no manejo.

Antes de o evento iniciar, 50 pessoas responderam ao questionário, a idade média foi de 29 anos, mínimo de 19 anos e máximo de 61 anos. Ao final do evento, após a parte prática, 
35 pessoas responderam ao questionário. A idade média foi de 31,1 anos, mínimo de 20 anos e máximo de 42 anos.

A tabela 1 mostra a caracterização dos serviços de saúde de acordo com instrumento avaliativo antes e após o término do evento.

Tabela 1- Distribuição da caracterização dos serviços de saúde de acordo com instrumento avaliativo do evento. Diamantina, 2017

\begin{tabular}{|c|c|c|c|c|}
\hline \multirow[t]{2}{*}{ Caracterização dos serviços de saúde } & \multicolumn{2}{|c|}{ Antes do Evento } & \multicolumn{2}{|c|}{ Após Término } \\
\hline & $\mathrm{n}$ & $\%$ & $\mathrm{n}$ & $\%$ \\
\hline \multicolumn{5}{|l|}{ Municípios } \\
\hline Alvorada de Minas & 1 & 2,0 & - & - \\
\hline Araçuaí & 3 & 6,0 & 3 & 8,6 \\
\hline Berilo & - & - & 1 & 2,9 \\
\hline Capelinha & - & - & 3 & 8,6 \\
\hline Carbonita & 3 & 6,0 & 1 & 2,9 \\
\hline Coluna & 1 & 2,0 & 1 & 2,9 \\
\hline Diamantina & 18 & 36,0 & 3 & 8,6 \\
\hline Gouveia & 1 & 2,0 & - & - \\
\hline Itamarandiba & 4 & 8,0 & 6 & 17,1 \\
\hline Itaobim & - & - & 1 & 2,9 \\
\hline Minas Novas & 2 & 4,0 & 2 & 5,7 \\
\hline Presidente Kubiteschek & 2 & 4,0 & - & - \\
\hline S. G. Rio Preto & 1 & 2,0 & 1 & 2,9 \\
\hline Serro & 2 & 4,0 & - & - \\
\hline Turmalina & 1 & 2,0 & 2 & 5,7 \\
\hline Virgem da Lapa & 3 & 6,0 & 1 & 2,9 \\
\hline Não estão inseridos no serviço & 8 & 16,0 & - & - \\
\hline Perdas & - & - & 10 & 28,6 \\
\hline \multicolumn{5}{|l|}{ Possui Maternidade } \\
\hline Sim & 33 & 66,0 & 23 & 65,7 \\
\hline Não & 13 & 26,0 & 8 & 22,9 \\
\hline Perdas & 4 & 8,0 & 4 & 11,4 \\
\hline \multicolumn{5}{|l|}{ Profissão } \\
\hline Enfermeiro & 22 & 44,0 & 21 & 60,0 \\
\hline Médico & 9 & 18,0 & 10 & 28,6 \\
\hline Estudante Enfermagem & 4 & 8,0 & - & - \\
\hline Estudante Medicina & 15 & 30,0 & 4 & 11,4 \\
\hline \multicolumn{5}{|l|}{ Realiza Partos? } \\
\hline Sim & 20 & 40,0 & 18 & 51,4 \\
\hline Não & 23 & 46,0 & 14 & 40,0 \\
\hline Perdas & 7 & 14,0 & 3 & 8,6 \\
\hline
\end{tabular}

Revista Extensão em Foco, nº 16, Jul./ Set. (2018), p. 01 - 11. 


\begin{tabular}{|c|c|c|c|c|}
\hline Sim & 32 & 64,0 & 22 & 62,9 \\
\hline Não & 5 & 10,0 & 7 & 20,0 \\
\hline Perdas & 13 & 26,0 & 6 & 17,1 \\
\hline \multicolumn{5}{|c|}{$\begin{array}{l}\text { Diferença entre os atendimentos de } \\
\text { pacientes do SUS e convênio }\end{array}$} \\
\hline Sim & 6 & 12,0 & 5 & 14,3 \\
\hline Não & 23 & 46,0 & 13 & 37,1 \\
\hline Perdas & 21 & 42,0 & 17 & 48,6 \\
\hline \multicolumn{5}{|c|}{ Partograma Implantado } \\
\hline Sim & 27 & 54,0 & 16 & 45,7 \\
\hline Não & 11 & 22,0 & 14 & 40,0 \\
\hline Perdas & 12 & 24,0 & 5 & 14,3 \\
\hline \multicolumn{5}{|c|}{$\begin{array}{l}\text { É oferecido métodos não farmacológicos } \\
\text { de alívio da dor? }\end{array}$} \\
\hline Sim & 26 & 52,0 & 18 & 51,4 \\
\hline Não & 13 & 26,0 & 12 & 34,3 \\
\hline Perdas & 11 & 22,0 & 5 & 14,3 \\
\hline \multicolumn{5}{|c|}{$\begin{array}{l}\text { O contato pele a pele é feito após quantos } \\
\text { minutos de vida? }\end{array}$} \\
\hline Imediato & 15 & 30,0 & 12 & 34,3 \\
\hline 1 minuto & 6 & 12,0 & 3 & 8,6 \\
\hline 2 a 5 minutos & 4 & 8,0 & 1 & 2,9 \\
\hline 10 minutos & 3 & 6,0 & 1 & 2,9 \\
\hline 15 a 20 minutos & 1 & 2,0 & - & - \\
\hline 60 minutos & 1 & 2,0 & - & - \\
\hline Não é feito & 2 & 4,0 & 5 & 14,3 \\
\hline Perdas & & & 13 & 37,1 \\
\hline \multicolumn{5}{|c|}{$\begin{array}{l}\text { O cordão umbilical é cortado após quanto } \\
\text { tempo de vida? }\end{array}$} \\
\hline Imediato & 2 & 4,0 & 3 & 8,6 \\
\hline 1 a 3 minuto & 29 & 92,0 & 24 & 68,4 \\
\hline 5 minutos & 2 & 4,0 & 1 & 2,9 \\
\hline Perdas & & & 7 & 20,0 \\
\hline \multicolumn{5}{|c|}{$\begin{array}{l}\text { É realizado o manejo ativo no terceiro } \\
\text { estágio? }\end{array}$} \\
\hline $\operatorname{Sim}$ & 21 & 42,0 & 23 & 65,7 \\
\hline Não & 6 & 12,0 & 2 & 5,7 \\
\hline Perdas & 23 & 46,0 & 10 & 28,6 \\
\hline
\end{tabular}

Fonte: dados coletados durante o evento.

Com base nas respostas ao questionário foi percebido que, apesar de garantido às parturientes o direito à presença de acompanhante durante o trabalho de parto, parto e pósparto imediato, resguardado pela Lei $\mathrm{n}^{\circ}$ 11.108, de 7 de abril de 2005 (BRASIL, 2005), o mesmo ainda não foi implementado em Coluna, Itamarandiba e Itaobim. 
Com relação à diferença de atendimento entre o SUS e o convênio observa-se melhores instalações físicas, com presença de quarto privativo e maior conforto para a parturiente e família do convênio. Em alguns locais foi relatada uma maior atenção da equipe médica aos pacientes de convênio.

Em relação ao partograma ele não foi implantado em Berilo, Itamarandiba, Itaobim, Minas Novas, São Gonçalo do Rio Preto e Virgem da Lapa. Os participantes de Berilo e Virgem da Lapa responderam que realizam poucos partos na cidade.

Entre os métodos não farmacológicos para alívio da dor, os mais descritos foram a bola, deambulação e massagem.

A Diretriz Nacional de Assistência ao Parto Normal recomenda para o recém-nascido, a termo e saudável, o contato pele a pele de forma imediata, ou seja, logo após o nascimento e por um período de uma hora. As instituições que ainda não fazem devem implantá-lo com o intuito de estimular o aleitamento materno na primeira hora de vida, aumentar o vínculo mãefilho e garantir calor para o RN (BRASIL, 2016).

A Diretriz citada acima recomenda realizar o clampeamento do cordão umbilical entre 1 a 5 minutos ou de forma fisiológica quando cessar a pulsação, exceto se houver alguma contra indicação em relação ao cordão ou necessidade de reanimação neonatal (BRASIL, 2016). Observa-se que uma maioria respondeu realizar esta prática entre 1 a 3 minutos, prática recomendada pelas evidências científicas.

O manejo ativo também foi referido pela maioria dos participantes e inclui a prática de: uso rotineiro de drogas uterotônicas (ocitocina), clampeamento e secção precoce do cordão umbilical, tração controlada do cordão após sinais de separação placentária (BRASIL, 2016).

Para avaliar o evento foram realizadas perguntas para verificar o nível de conhecimento dos participantes antes do evento.

Se comparado ao primeiro questionário dado, houve um aumento das respostas corretas e diminuição das perguntas deixadas sem responder (perdas). É importante enfatizar a 
importância de uma educação continuada já que memorizar nome do medicamento e dosagem não é algo simples.

A partir deste trabalho foi possível perceber a visível falta de recurso mínimo para atender urgência materna nos municípios. Foi relatado indisponibilidade de medicações para tocólise e sepse. Sugere-se como extensão deste trabalho um levantamento mais minucioso dos medicamentos imprescindíveis para atendimento de uma emergência. A proposta é retornar na reunião dos gestores e levar mais esta demanda. Sabe-se que o país passa por dificuldades financeiras, porém, como o quantitativo de partos é pequeno nestes municípios sugere-se a aquisição de um quantitativo menor de medicamentos.

Os avaliadores do evento consideraram positivo a entrega do protocolo de condutas clínicas em um CD já que possibilita que as pessoas consultem quando tiver dúvida.

\section{Conclusão}

A proposta desta intervenção é desenvolver estratégias de combate a mortalidade materna. Sabe-se que ações pontuais podem não ter êxito se não houver uma continuidade, porém entende-se que diversas estratégias foram utilizadas na tentativa de reduzir a mortalidade materna como a atualização e divulgação de um protocolo de condutas clínicas, entrega de check list de condutas e insumos de materiais para atender hemorragia e eclampsia, capacitação teórico-prática, dentre outras. Profissionais capacitados e recursos disponíveis na instituição são imprescindíveis para a redução da mortalidade materna.

Sabe-se que a adesão da equipe para basear sua prática clínica em um protocolo de condutas não será realizada por todos, porém acreditamos na sensibilização constante dos profissionais e na importância de uma prática homogênea baseada em evidência. No dia a dia as condutas de profissionais tendem a contagiar os mais resistentes a mudança. Este processo leva tempo, por isto caberá aos profissionais dos hospitais a capacitação em serviço, oportunizar momentos de aprendizado durante o plantão, atualizar o protocolo a cada ano e assim por diante.

Revista Extensão em Foco, n 16, Jul./ Set. (2018), p. 01 - 11. 
Espera-se que grandes atores estratégicos tenham sido sensibilizados e que mudança da pratica obstétrica aconteça. Torna-se necessário incentivar outros eventos desta natureza para que a mortalidade materna na região seja diminuída cada vez mais.

\section{REFERÊNCIAS}

BRASIL. Lei $\mathbf{n}^{\mathbf{0}}$ 11.108, de 7 de abril de 2005. Altera a Lei no 8.080, de 19 de setembro de 1990, para garantir às parturientes o direito à presença de acompanhante durante o trabalho de parto, parto e pós-parto imediato, no âmbito do Sistema Único de Saúde - SUS. Disponível em:< http://www.planalto.gov.br/ccivil_03/_ato2004-2006/2005/lei/111108.htm>. Acesso em: 09 de ago. 2017.

BRASIL. Diretriz Nacional de Assistência ao Parto Normal. Comissão Nacional de Incorporação de Tecnologias no SUS, 2016.

DIAS, J.A. Protocolo de Condutas Clínicas. Protocolos Clínicos do Departamento de Obstetrícia e Ginecologia. Hospital Nossa Senhora da Saúde: Diamantina, 2017.

DIAS, J.A. Estudo da mortalidade materna na região do Alto Jequitinhonha. Dissertação apresentada ao Programa de Mestrado Ensino em Saúde. Minas Gerais: Diamantina, 2016.

HNSS. Hospital Nossa Senhora da Saúde. Disponível em: < http://www.hnss.org.br/>. Acesso em: 25 de out. 2017.

ONUBR. Conheça os novos 17 Objetivos de Desenvolvimento Sustentável da ONU. Disponível em:<https://nacoesunidas.org/conheca-os-novos-17-objetivos-dedesenvolvimento-sustentavel-da-onu/>. Acesso em: 11 ago 2017.

SANCHES, N.C; MAMEDE, F.V; VIVANCOS, R.B.Z. Perfil das mulheres submetidas à cesareana e assistência obstétrica na maternidade pública em Ribeirão Preto. Texto Contexto Enferm. v.21, n.2, p. 418-26, 2012 apud DIAS, J.A. Estudo da mortalidade materna na região do Alto Jequitinhonha. Dissertação apresentada ao Programa de Mestrado Ensino em Saúde. Minas Gerais: Diamantina, 2016.

SOUZA, J.P. A mortalidade materna e os novos objetivos de desenvolvimento sustentável (2016-2030). Rev Bras Ginecol Obstet. v.37, n.12, p.549-51, 2015. 